\title{
Erratum to: Fatal pleural mesothelioma in Japan (2003-2008): evaluation of computed tomography findings
}

\author{
Katsuya Kato ${ }^{1,6} \cdot K^{2}$ ichi Gemba ${ }^{2,7} \cdot$ Nobukazu Fujimoto $^{2} \cdot$ Keisuke Aoe $^{3}$. \\ Yukio Takeshima $^{4} \cdot$ Kouki Inai $^{4,8} \cdot$ Takumi Kishimoto $^{5}$
}

Published online: 7 April 2016

(C) Japan Radiological Society 2016

\section{Erratum to: Jpn J Radiol DOI 10.1007/s11604-016-0539-1}

The original version of the article cited above unfortunately contained an error. The "Ethical statement" section should read as follows:

Ethical statement This study was conducted according to the Ethical Guidelines for Clinical Research by the
Japanese Ministry of Health, Labor and Welfare. This study was approved by the Japan Health, Labor, and Welfare Organization and the ethical review boards of the principal investigator's institution (Okayama Rosai Hospital). Patient confidentiality was strictly maintained. Informed consent was obtained from the closest living relatives of each patient.
The online version of the original article can be found under doi:10.1007/s11604-016-0539-1.

Katsuya Kato

kato-rad@med.kawasaki-m.ac.jp

1 Department of Radiology, Okayama University Hospital, 2-1-1 Shikatacho, Okayama 7008558, Japan

2 Department of Medical Oncology, Okayama Rosai Hospital, 1-10-25 Chikkomidorimachi, Okayama 7028055, Japan

3 Department of Medical Oncology, National Hospital Organization Yamaguchi-Ube Medical Center, 685 Higashikiwa, Ube 7550241, Japan

4 Department of Pathology, Hiroshima University Graduate School of Medicine, 1-2-3 Kasumi, Hiroshima 7340037, Japan
5 Department of Internal Medicine, Okayama Rosai Hospital, 1-10-25 Chikkomidorimachi, Okayama 7028055, Japan

6 Present Address: Department of Diagnostic Radiology 2, Kawasaki Medical School, 2-1-80 Nakasange, Kita-ku, Okayama 7008505, Japan

7 Present Address: Department of Respiratory Medicine, Chugoku Chuo Hospital, 148-13 Miyukicho Oaza, Kamiiwanari, Fukuyama 7200001, Japan

8 Present Address: Pathologic Diagnostic Center, Inc., 11-28 Hacchobori, Naka-ku, Hiroshima 7300013, Japan 